\title{
Processed human amniotic fluid retains its antibacterial activity
}

\author{
Yong Mao ${ }^{1}$, Jan Pierce ${ }^{2,3}$, Anya Singh-Varma', Michael Boyer ${ }^{2,3}$, Joachim Kohn ${ }^{1}$ and Jo-Anna Reems ${ }^{2,3^{*}}$
}

\begin{abstract}
Background: Human amniotic fluid (AF) contains numerous nutrients, trophic factors and defense proteins that provide a nurturing and protective environment for fetal development. Based on reports that AF has antibacterial, anti-inflammatory and regenerative properties, we designed a novel method to process AF for use in clinical care.

Methods: Six randomly selected lots of processed AF (PAF) were examined to determine whether they retained their antibacterial activity against a panel of wound-associated pathogens E. faecium, S. aureus, K. pneumoniae, A. baumannii, P. aeruginosa, and E. aerogenes (ESKAPE). To identify proteins in PAF that might be responsible for its antibacterial activity, three different lots of pAF were analyzed with quantitative cytokine arrays that consisted of 400 unique human proteins. One protein identified by microarrays, lactoferrin, and a second prominent antibacterial protein that was not identified by microarrays, lysozyme, were examined by depletion experiments to determine their contribution to the antibacterial activity of PAF.

Results: All six lots of pAF exhibited antibacterial activity against ESKAPE microorganisms, especially against the pathogens predominately found in chronic wounds (i.e. S. aureus and P. aeruginosa). Thirty-one of the peptides on the microarray were annotated as having antibacterial activity and 26 of these were detected in PAF. Cystatin C and lactoferrin were among the most highly expressed antibacterial proteins in PAF. Cystatin C and lactoferrin were confirmed by ELISA to be present in PAF along with lysozyme. Immunoprecipitation of lactoferrin and lysozyme reduced, but did not abolish the antibacterial activities of PAF.
\end{abstract}

Conclusion: Our data demonstrate that pAF maintains antibacterial activity via the preservation of antibacterial proteins against a broad spectrum of wound-associated pathogens.

Keywords: Amniotic fluid, Antibacterial, ESKAPE, Immunoprecipitation

\section{Background}

The first use of human placental membranes for the treatment of diseases occurred with Chinese and Japanese traditional medicines centuries ago, and around the turn of the 20th century for Western cultures [1,2]. Since these early applications, investigators identified that there are antibacterial and other paracrine factors in amniotic membrane that modulate the wound healing process $[1$, 3]. Like amniotic membrane, amniotic fluid (AF) plays important roles in the development and the protection of the fetus [4]. One property of AF that helps to defend the

\footnotetext{
*Correspondence: joanna.reems@hsc.utah.edu

${ }^{3}$ Cell Therapy and Regenerative Medicine Facility, University of Utah, 676

Arapeen Drive, Suite 300, Salt Lake City, UT 84108, USA

Full list of author information is available at the end of the article
}

fetus against pathogens is its antibacterial activity [5-7]. AF contains numerous defense proteins and cytokines and antibacterial peptides, like cystatin $\mathrm{C}$, lactoferrin and lysozyme [8-11]. Other components that are present in AF that confer antibacterial activity include transferrin, $\beta$-lysin, peroxidases, immunoglobulins, and zinc-peptide complexes $[8,12-14]$. Also, chemokines such as CXCL1 and CXCL14 with known antibacterial activities are present in AF [15].

Amniotic fluid is a complex matrix that can be viewed as containing multiple fractions: (1) an insoluble fraction that consists of components like lanugo, vernix and cellular elements; (2) a soluble fraction that is made up of carbohydrates, proteins, lipids, electrolytes and metabolites and; (3) a fraction that contains extracellular vesicles 
(ECVs). Based on the knowledge that cytokines, growth factors and chemokines play major roles in activating endogenous mechanisms to facilitate repair and regeneration, we developed a novel approach to produce a processed AF (pAF) that eliminates a majority of its insoluble components (i.e. lanugo, vernix and cells) [16]. Interestingly, despite the removal of these insoluble components that include the stem cells, pAF supports the healing of chronic and acute wounds (unpublished observation). This observation suggests that active ingredients retained within the soluble fraction of pAF have properties capable of facilitating wound healing. From a previous study, we reported that $76 \%$ of a total of 400 proteins that we tested were present in pAF and that a majority of these proteins had roles in host defense [16]. Among the host defense peptides are proteins known for their role in the inflammatory response, innate immunity, immune modulation, and/or as having antibacterial activity.

Chronic wound environments are often colonized with microbes [17] and this colonization by microorganisms likely results in delayed healing due to infection [18, 19]. Delays in the healing process for chronic wounds is also attributable to prolonged inflammation caused by a lack of proper growth factors and cytokines [20]. Consequently, therapies that have antibacterial and antiinflammatory activity would be a good choice for treating wounds. Recognizing that pAF facilitates the healing of chronic and acute wounds, the goals of this study were to assess the antibacterial activity of unprocessed AF relative to $\mathrm{pAF}$, to evaluate the antibacterial activity of pAF from different donors, and to begin to characterize the role of specific antibacterial proteins in pAF against a panel of wound-associated pathogens.

\section{Materials and methods}

\section{Collection and processing of amniotic fluid}

Donor consent, screening and infectious disease testing of amniotic fluid (AF) were previously described [16]. The collection and processing of AF were also previously described [16]. Prior to processing AF, several aliquots were removed and stored frozen at $-80^{\circ} \mathrm{C}$ (i.e. pre-processed AF). Upon the completion of the final processing step, aliquots of pAF were removed and the samples were maintained at $-80{ }^{\circ} \mathrm{C}$ until the time of use.

\section{Bacterial culture and preparation of inoculums}

Clinical isolates of ESKAPE bacterial strains were purchased from ATCC (Manassas, VA): E. faecium ATCC (B) $51559^{\mathrm{TM}}$, S. aureus $\mathrm{ATCC}^{\circledR} 25923^{\mathrm{TM}}$, K. pneumoniae $\mathrm{ATCC}^{\circledR} 700603$, A. baumannii $\mathrm{ATCC}^{\circledR} 49466^{\mathrm{TM}}$, P. aeruginosa $\mathrm{ATCC}^{\circledR} 15692^{\mathrm{TM}}$, and E. aerogenes $\mathrm{ATCC}^{\circledR} 49469^{\mathrm{TM}}$. All bacterial strains were cultured and maintained as instructed by $\mathrm{ATCC}^{\circledR}$. Optimal culture media used are tryptic soy broth (TSB) for S. aureus, P. aeruginosa, $E$. aerogenes, $A$. baumannii, brain heart infusion (BHI) medium for E. faecium and nutrient broth for K. pneumoniae. Preparation of bacterial inoculum was performed as described previously [21]. Briefly, bacteria were cultured in their defined optimal culture medium at $37^{\circ} \mathrm{C}$ with shaking until optical density reached 0.2 to 0.6 at $600 \mathrm{~nm}$ $\left(\mathrm{OD}_{600}\right)$. The number of colony forming units (CFUs) for each strain was estimated based on an $\mathrm{OD}_{600}=1.0$, which corresponds to $10^{9} \mathrm{CFU} / \mathrm{mL}$. To prepare the inoculum for antibacterial assays, the bacterial stocks were serially diluted with culture medium or pAF to approximately $1 \times 10^{3} \mathrm{CFU} / \mathrm{mL}$ of bacteria. For each experiment, the actual CFU of each inoculum was determined by preparing serial dilutions and then plating onto TSB agar plates (BD, Franklin Lakes, NJ).

\section{Inhibition of bacterial growth by pAF}

An inoculum of $P$. aeruginosa or $S$. aureus $\left(1 \times 10^{3}\right.$ $\mathrm{CFU} / \mathrm{mL}$ ) was added to $\mathrm{pAF}$ that had been prepared by diluting pAF in TSB. The cultures were incubated at $37{ }^{\circ} \mathrm{C}$ for $24 \mathrm{~h}$. The growth of bacteria was monitored using an alamarBlue assay (ThermoFisher Scientifics; Waltham, MA) by following the manufacture's protocol. The fluorescent intensity was measured using a TECAN Spark $10 \mathrm{M}$ plate reader (TECAN, Morrisville NC) at Ex560 nm/Em590 nm. The growth of bacteria in the presence of pAF was normalized to the growth in the absence of pAF.

\section{Quantification of bacterial growth in the presence of pAF}

Bacterial growth was assessed as described with modifications [22]. Each strain of bacteria $\left(1 \times 10^{3} \mathrm{CFU} /\right.$ $\mathrm{mL}$ ) was added to $1 \mathrm{~mL}$ of AF or $1 \mathrm{~mL}$ optimal culture medium. The cultures were incubated at $37{ }^{\circ} \mathrm{C}$ with shaking for $24 \mathrm{~h}$. Serial dilutions were then prepared for each culture and plated onto TSB agar plates. CFUs were counted after overnight incubation at $37{ }^{\circ} \mathrm{C}$. The antibacterial activity of pAF was expressed in log reductions, which was calculated as the $\log _{10} \mathrm{CFU}$ (Control) $-\log _{10} \mathrm{CFU}$ (pAF). Data from two independent experiments $(\mathrm{n}=3$ for each experiment) were pooled together to calculate the mean and standard deviation.

\section{Protein array}

Quantitative Protein arrays were performed as previously described [16]. Briefly, pAF from three maternal collections were sent to RayBiotech to simultaneously and quantitatively measure the concentration of 400 human cytokines using the Quantibody ${ }^{\circledR}$ Human Cytokine Antibody Array 9000 (RayBiotech, In., Norcross, GA). Controls and serial dilutions of cytokine standards were prepared according to the manufacturer's instructions 
and were added to chip wells. After processing the chips according to the manufacturer's instructions, the chips were analyzed using the Quantibody ${ }^{\circledR}$ Q-Analyzer software (RayBiotech, Inc.). Proteins were classified according to their biological function by surveying the Human Protein Reference Database (http://www.hprd.org/ index_html), Cytokines \& Cells Online Pathfinder Encyclopedia (COPE) http://www.copewithcytokines.de/), GeneCards $^{\circledR}$ (http://www.genecards.org/), and the biomedical literature in PubMed (http://www.ncbi.nlm.nih. gov/pubmed).

\section{Detection of human lysozyme, cystatin C and lactoferrin in PAF using ELISA}

The presence of lysozyme, cystatin $C$ and lactoferrin from different lots of pAF was quantified using quantitative sandwich ELISA assays according to the manufacturer's instructions (Abcam, Cambridge, MA): Lysozyme (Human Lysozyme ELISA kit ab108880), Lactoferrin (Human Lysozyme ELISA kit ab200015), and cystatin C (Human Lysozyme ELISA kit ab179883).

\section{Immunoprecipitation (IP)}

Selective depletion of lysozyme and lactoferrin from AF was accomplished by immunoprecipitation (IP) as described [22]. Briefly, rabbit polyclonal anti-lysozyme antibody (ab2408) and anti-lactoferrin antibody (ab15811) were purchased from Abcam. Anti-lysozyme antibodies $(15 \mu \mathrm{g} / \mathrm{mL})$ and anti-lactoferrin antibodies $(4 \mu \mathrm{g} / \mathrm{mL})$ were added individually (IP) or together (CoIP) to $1 \mathrm{~mL}$ of AF. As a control, the same volume of PBS was added to $1 \mathrm{~mL}$ of AF. The samples were mixed at $4{ }^{\circ} \mathrm{C}$ for overnight. A $50 \mu \mathrm{L}$ slurry of Protein A/G agarose plus resin: sc-2003 (Santa Cruz Biotechnology; Dallas, TX) was washed twice with $1 \mathrm{~mL}$ of PBS and mixed with each pAF with or without antibodies for $4 \mathrm{~h}$ at $4{ }^{\circ} \mathrm{C}$. Mixtures were then centrifuged at $2000 \mathrm{rpm}$ for $3 \mathrm{~min}$ and resulting supernatants were transferred to individual bacterial culture tubes. Inoculums of $1 \times 10^{3} \mathrm{CFU}$ of $P$. aeruginosa or $S$. aureus were added to each culture tube and incubated with shaking at $37{ }^{\circ} \mathrm{C}$. After $24 \mathrm{~h}$, CFUs for each culture were quantified by serial dilution plating as previously described [22].

\section{Statistical analysis}

Each independent experiment contained 3 or more biological repeat samples $(n \geq 3)$, and data is presented as the mean \pm standard deviation. One-way ANOVA with a Tukey's multiple comparisons test was performed to determine statistical significance. Differences were considered significant at a $\mathrm{p}$ value of $<0.05$.

\section{Results}

Processed AF inhibited the growth of P. aeruginosa and $S$. aureus

The antibacterial activities of unprocessed and processed AF were determined by measuring the growth of $P$. aeruginosa or S. aureus in the presence of either pre-processed AF or post-processed AF (Fig. 1). With increasing concentrations of unprocessed $\mathrm{AF}$ and $\mathrm{pAF}$, the growth of either P. aeruginosa (Fig. 1a) or S. aureus (Fig. 1b) decreased $70 \%$ or $90 \%$, respectively.

Next, we examined the antibacterial activity of pAF from six different maternal collections against $P$. aeruginosa (Fig. 2a) and S. aureus (Fig. 2b). As was evident by a reduction in bacterial growth for both microorganisms, all six lots of pAF showed an antibacterial effect against P. aeruginosa (Fig. 2a) and S. aureus (Fig. 2b). Distinct
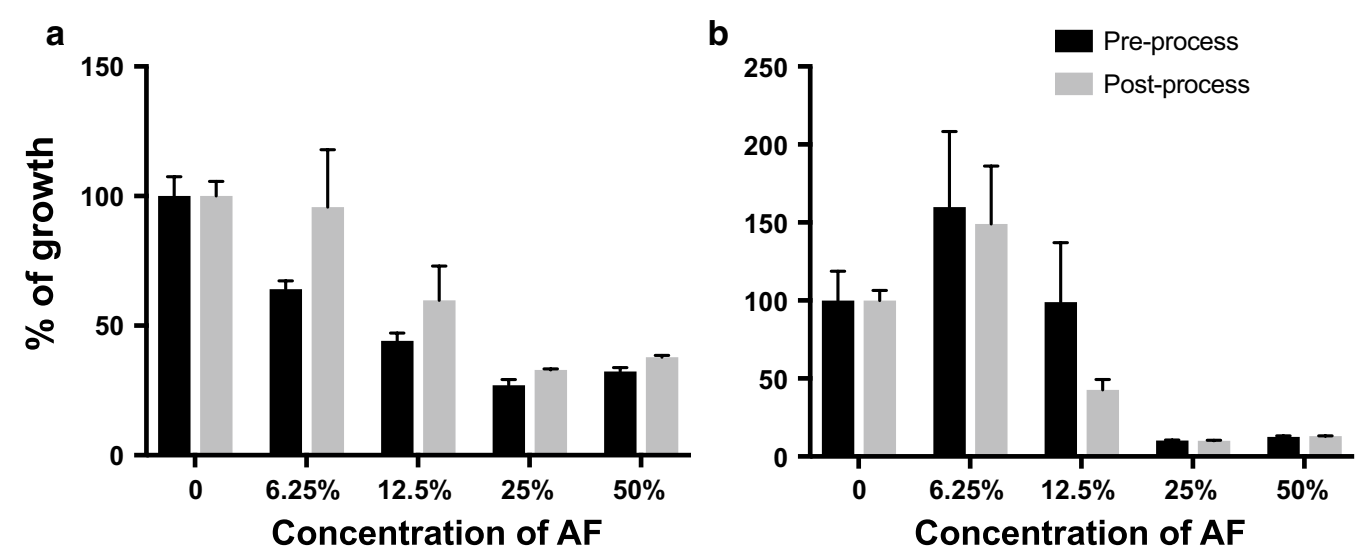

Fig. 1 Relative antibacterial activity of AF before and after processing. The growth of P. aeruginosa (a) or S. aureus (b) in tryptic soy broth without AF or in TSB with different concentrations of AF were quantified using alamarBlue assay. The percent of growth reduction with AF/pAF is calculated as: fluorescent intensity of culture with AF/fluorescent intensity of control (no AF) $\times 100$. Data are presented as mean $\pm S D(n=3)$ 

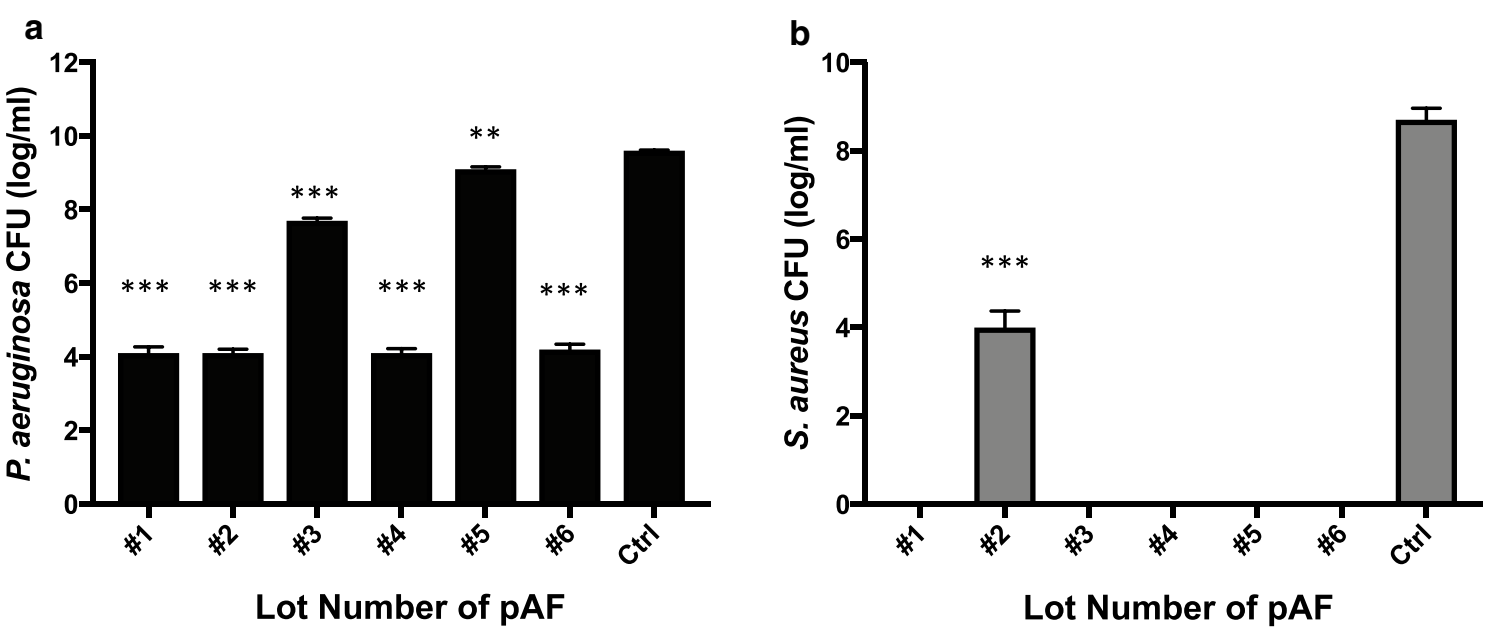

Fig. 2 Antibacterial activity of processed AF. The growth of P. aeruginosa (a) or S. aureus (b) in different lots of pAF, PBS or were quantified by serial dilution and CFU counting after $24 \mathrm{~h}$ culture. Data are presented as mean \pm SD for each lot $(n=3)$. Statistical analysis was performed to compare each lot of pAF with Control (Ctrl). ${ }^{* *} p<0.01$ and ${ }^{* * *} p<0.005$

Table 1 Antibacterial activity of processed AF against ESKAPE pathogens

\begin{tabular}{|c|c|c|c|c|c|c|}
\hline Lot \# & E. aerogenes & S. aureus & K. pneumoniae & A. baumannii & P. aeruginosa & E. faecium \\
\hline 1 & $7.2 \pm 0.1$ & $8.7 \pm 0.0$ & $5.5 \pm 0.1$ & $3.3 \pm 0.1$ & $5.5 \pm 0.2$ & $2.3 \pm 0.2$ \\
\hline 2 & $6.8 \pm 0.0$ & $4.7 \pm 0.4$ & $4.8 \pm 0.3$ & $5.2 \pm 0.1$ & $5.5 \pm 0.1$ & $2.6 \pm 0.1$ \\
\hline 3 & $7.7 \pm 0.6$ & $8.7 \pm 0.0$ & $0.9 \pm 0.0$ & $4.8 \pm 0.1$ & $2.0 \pm 0.1$ & $9.2 \pm 0.0$ \\
\hline 4 & $7.5 \pm 0.1$ & $8.7 \pm 0.0$ & $4.8 \pm 0.7$ & $3.7 \pm 0.1$ & $5.5 \pm 0.1$ & $2.4 \pm 0.1$ \\
\hline 5 & $0.7 \pm 0.1$ & $8.7 \pm 0.0$ & $0.6 \pm 0.1$ & $0.8 \pm 0.0$ & $0.5 \pm 0.1$ & $1.8 \pm 0.1$ \\
\hline 6 & $7.6 \pm 0.2$ & $8.7 \pm 0.0$ & $4.4 \pm 0.4$ & $5.0 \pm 0.1$ & $5.4 \pm 0.1$ & $3.0 \pm 0.2$ \\
\hline
\end{tabular}

Expressed as a log reduction in growth against each bacterium; Log reduction $=\log _{10} \mathrm{CFU}_{(\mathrm{Ctrl})}-\log _{10} \mathrm{CFU}_{(\mathrm{AF})}$

lots of pAF appeared to have differential activity against different bacteria. Four out of six lots of pAF showed a growth inhibition of $P$. aeruginosa over 5 logs and two lots showed inhibition of less than 2 logs. On the other hand, five out of six lots showed a bactericidal effect against $S$. aureus (Fig. 2b).

\section{Processed AF has antibacterial activity against ESKAPE pathogens}

After establishing that pAF retained the antibacterial activity of unprocessed AF, we examined the activity of pAF against a panel of Gram-positive and Gramnegative microorganisms that are known for their association with wound microenvironments and their tendency to have an antibiotic resistance nature. This panel of microbes includes: E. faecium, S. aureus, $K$. pneumoniae, A. baumannii, $P$. aeruginosa, and E. aerogenes and is often referred as the ESKAPE panel [23]. We quantified the growth of ESKAPE microbes in the presence of multiple lots of pAF (Table 1). The growth of each strain of bacteria in the presence of pAF was compared with the growth in optimal culture medium. Besides the inhibitory effects of pAF on the growth of $S$. aureus and $P$. aeruginosa as described in Fig. 2, all six randomly selected lots of pAF (lots 1-6) showed antibacterial activity against E. aerogenes. Among them, five lots of pAF showed strong activity against $E$. aerogenes (over 7 logs of growth reduction) and one lot (i.e. lot 5) had a lower activity against $E$. aerogenes. Four out of six lots reduced the growth of $K$. pneumoniae for over $4 \operatorname{logs}$ while lots 3 and 5 of pAF showed weaker inhibitory activity against $K$. pneumonia. A. baumannii showed over 3 logs of growth reduction in the presence of all six lots of pAF except for lot 5 , which had a weaker activity. The activities against $E$. faecium were comparable among different lots of pAF except for lot 3, which showed bactericidal effects against E. faecium. These results indicate that pAF has antibacterial activity and the antibacterial activity does vary by lot and by organism. 


\section{Antibacterial proteins/peptides in AF}

To evaluate potential proteins with antibacterial properties in pAF, we focused on antibacterial proteins that we identified from comprehensive protein array data sets that we previously published [16] (Fig. 3). The proteins identified were from 4 replicates of pAF from 3 different lots that were not used for the antibacterial testing of this study. Of 400 proteins tested, an average of $304 \pm 20$ (average \pm SD) peptides were detected in the three lots of pAF. Within the list of 400 proteins tested, 31 peptides were annotated as possessing antibacterial properties. Of these 31 proteins tested, 26 or $84 \%$ of the proteins tested were present in pAF and 5 were not detectable. The results of the array showed that cystatin $C(511 \pm 122 \mathrm{ng} /$ $\mathrm{mL})$ and lactoferrin $(52.7 \pm 1.9 \mathrm{ng} / \mathrm{mL})$ stood out as having the highest quantitative levels among antibacterial proteins $(n=3$, mean $\pm S D)$. Also, of the 26 detectable antibacterial peptides, 17 of them were classified as chemokines (Fig. 3).

In the next set of experiments, we validated the results of the array data by quantifying the levels of cystatin $C$ and lactoferrin in pAF by using ELISA. We also selected to quantitate lysozyme levels in pAF as it had been previously reported by others to be present at high levels in $\mathrm{AF}$ and to confer antibacterial activity in AF [24]. The levels of lysozyme, cystatin $C$ and lactoferrin in six different lots of pAF were quantified using ELISA (Fig. 4). Among the different lots of pAF, the levels of lysozyme, cystatin $\mathrm{C}$, and lactoferrin varied. Three (i.e. lots 1,2 and 4) of the four lots (i.e. lots 1, 2, 4, and 6) of pAF that had stronger antibacterial activity against $P$. aeruginosa (Fig. 2) and K. pneumonia also showed higher levels of lysozyme (Fig. 4a). This observation indicates that $P$. aeruginosa and $K$. pneumoniae may be sensitive to lysozyme in pAF. No apparent correlation between lysozyme levels (Fig. 4a) and antibacterial activity were noted against $S$. aureus (Fig. 2b). Levels of cystatin C were relatively comparable among the different lots of pAF with the exception of lot 4, which showed the highest level of cystatin $C$ (Fig. 4b). No apparent correlation between cystatin C levels (Fig. 4b) and antibacterial activity were noted against $S$. aureus or P. aeruginosa (Fig. 2a, b). The levels of lactoferrin in different lots of pAF (Fig. 4c) showed a strong correlation with antibacterial activity against $S$. aureus among all six lots (Fig. 2b), where lot 2 with the lowest lactoferrin showed the lowest activity against $S$. aureus. No specific correlation was identified between the level of specific antibacterial protein and activity against $E$. aerogenes, A. baumannii or E. faecium. Nevertheless, we observed that the highest levels of antibacterial proteins (i.e. lots 1 and 4) correlated with highest overall antibacterial activities.

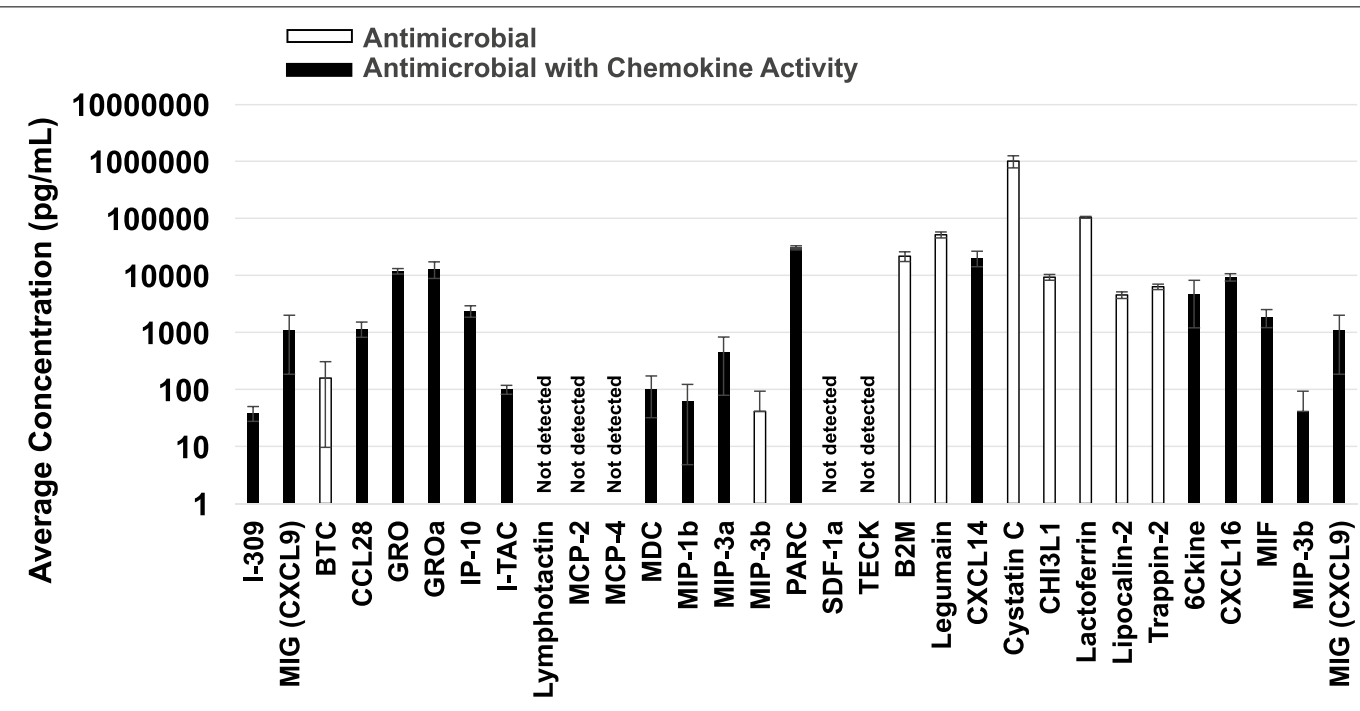

Peptide

Fig. 3 Proteins tested in pAF with known antibacterial activity. Quantibody ${ }^{\circledR}$ Human Cytokine Antibody Arrays 9000 were used to examine the presence or absence of 400 different peptides in pAF. Of the 400 peptides that were tested (i.e. three lots other than six lots examined for antibacterial activity), an average of $304 \pm 20$ proteins $(n=3)$ were positively identified in pAF. Thirty-one of the 400 proteins that were tested were known as antibacterial peptides. Of the 31 antibacterial proteins that were tested, 26 were present in pAF. Among the detectable antibacterial peptides, 17 of them are also classified as chemokines (black bars) and 9 of them are not chemokines (white bars) 

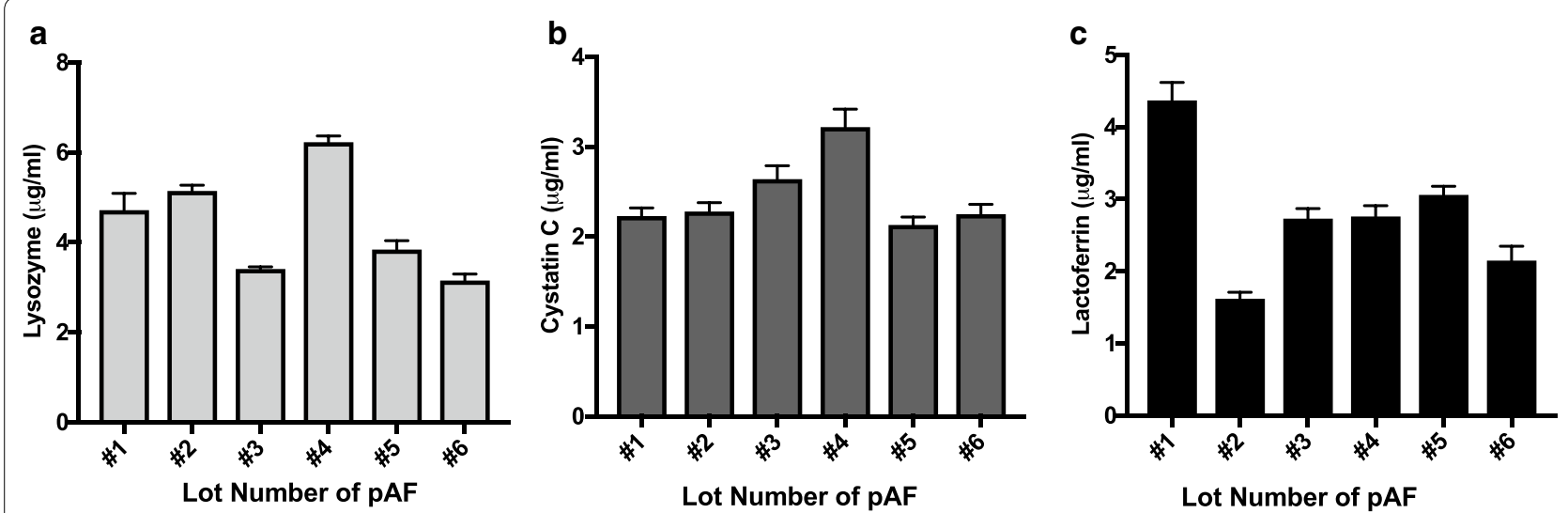

Fig. 4 The presence of antibacterial proteins in multiple lots of AF. The levels of lysozyme (a), cystatin C (b) or lactoferrin (c) in different lots of AF were quantified using ELISA as described in Materials and methods. Data are presented as mean $\pm S D(n=4)$

\section{Antibacterial proteins contribute to the antibacterial activity of PAF}

To evaluate the contribution of lactoferrin and lysozyme to the antibacterial activity of pAF, we selectively reduced the levels of these proteins in pAF by using immunoprecipitation (IP). Lot 2 was immunoprecipitated individually or in combination with antibodies against lactoferrin and with antibodies against lysozyme. As shown in Fig. 5 after IP with lactoferrin or lysozyme, the anti-P. aeruginosa activity of pAF was reduced by 4.9 - and 4.6-fold, respectively (Fig. 5a). However, no further reduction of anti-P. aeruginosa activity was observed after a Co-IP with lactoferrin and lysozyme. On the other hand, while the depletion of lysozyme did not reduce anti-S. aureus activity of pAF, IP with lactoferrin or Co-IP with lactoferrin and lysozyme reduced the anti-S. aureus activity of pAF by over 28 -fold (Fig. 5b).

\section{Discussion}

Reports of stem cells in amniotic fluid (AF) has attracted the efforts of a number of groups for developing commercial products of AF for the treatment of wounds and other therapeutic applications $[25,26]$. Based on our observation that there is a paucity of stem cells in AF and that expansion of the stem cells would be required to obtain therapeutic doses. We developed a processing

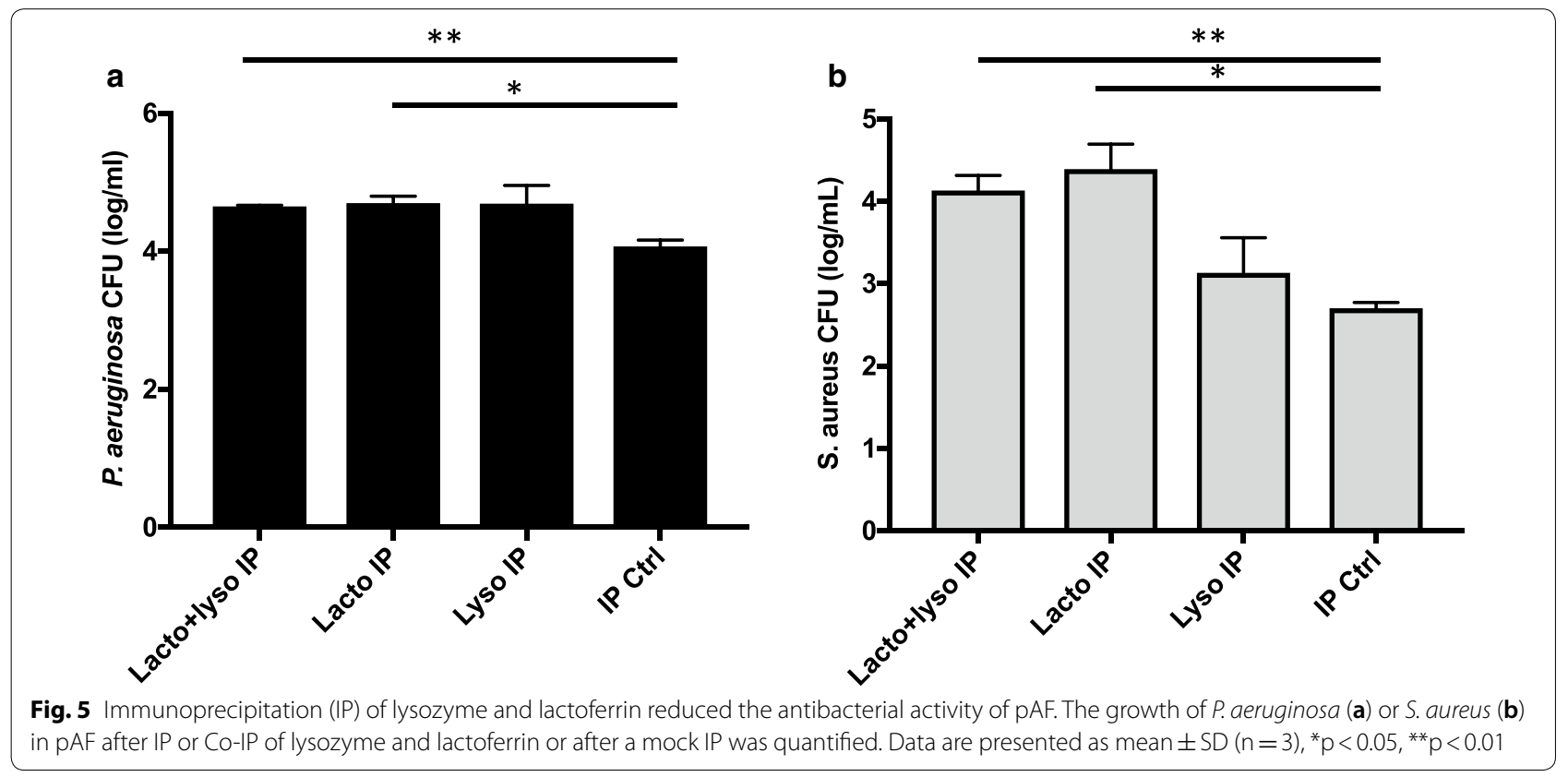


method that removes a majority of the insoluble components (i.e. cells, lanugo and vernix) while retaining soluble components (i.e. cytokines, growth factors, chemokines, antibacterial peptides) and extracellular vesicles [16]. Our formulation of pAF is based on the hypothesis that the soluble components and extracellular vesicles found in AF will activate endogenous mechanisms to facilitate immune responses, tissue repair and regenerative events.

Recognizing that delayed healing can occur when wounds are colonized with microbes and that a therapy with antibacterial activity is advantageous towards healing infected wounds, this study determined whether AF depleted of its insoluble components retained its antibacterial activity. We found that pAF maintains its antibacterial activity relative to unprocessed $\mathrm{AF}$ and that the soluble components in pAF are key contributors to its antibacterial activity. This study also shows that differences in the antibacterial activity of different lots of pAF exist against different pathogens (Table 1). Given that a standardized method is used to process AF, it is more likely that the differences in the antibacterial activity of different lots is due to inherent differences among donors rather than to the manufacturing strategy.

Of the 31 known anti-microbial proteins that we tested, 26 of them are present in pAF. Cystatin $C$ and lactoferrin were amongst the highest expressed antibacterial proteins that we tested. Cystatin $C$ not only had the highest quantitative levels among antibacterial proteins, but was also among the top 5 most highly expressed proteins of $\sim 300$ proteins that we identified and quantified in pAF [16]. Cystatin $C$ is a cysteine protease inhibitor [27] that is present in almost all tissue and body fluids and is reported to be a potent regulator of the inflammatory response. Cystatin $\mathrm{C}$ plays important roles in innate immunity by binding to components of the classical complement pathway and by modulating the actions of neutrophils via superoxide inhibition and chemotaxis [28-30]. Lactoferrin, is the most abundant protein in the whey fraction of human milk [31]. Lactoferrin plays a critical role in protecting the newborn infant from infection via its iron binding function that inhibits bacteria, fungus, viral and parasitic infections and by its antiinflammatory and immunomodulatory activities [32-34].

Seventeen of the antibacterial proteins that we identified in pAF also possess chemokine activity, a property that involves directing the immune response to sites of injury and infection. For example, PARC, the most highly expressed chemokine in pAF triggers lymphocyte responses, but not neutrophils [35]. While GRO and GROa attract neutrophils [36]. Others of the identified chemokines present in pAF are proteins that can attract monocytes, eosinophils, and even basophils. Moreover, some chemokines have also been shown to have antibacterial activity that directly interfere with infectious agents [15]. The significance of this finding is that different chemokines in pAF may recruit different types of white cells to sites of infection, inflammation and/or injury to promote repair of the site.

To determine whether a relationship could be established between the levels of antibacterial proteins and their activity against specific pathogens, we focused our effort on 3 proteins found in AF, lactoferrin, cystatin C and lysozyme. Knowing that lactoferrin has antibacterial activity against $S$. aureus [37] and lysozyme has lytic activity against $P$. aeruginosa over $S$. aureus $[24,38]$, we were particularly interested in whether the levels of these proteins correlated with the bactericidal activity of pAF. We found that of six pAF lots tested, all lots except for lot 2 showed bactericidal activity against $S$. aureus (Fig. $2 \mathrm{~b}$ ) and lot 2 also had the lowest levels of lactoferrin (Fig. 4c). Likewise, lower levels of lysozyme in lots 3 and 5 (Fig. 4a) corresponded with lower anti-P. aeruginosa activity (Table 1). However, lower levels of lysozyme activity for lot 6 did not correspond to lower anti-P. aeruginosa activity. Also, the levels of cystatin $C$, lysozyme and lactoferrin in lot 6 were not higher than that of lot 5 , but lot 6 had better overall antibacterial activity than lot 5 . These results suggest that lactoferrin and lysozyme levels may contribute to differential antibacterial activities of pAF. However, we did not identify one single protein of the 3 antibacterial proteins we tested whose level in AF determines the antibacterial activity of AF. This suggests that other proteins/peptides with antibacterial proteins in pAF are likely to contribute to pAF's antibacterial activity. Evidence that other antibacterial proteins in pAF are involved is supported by the results of our immunoprecipitation study. By specifically depleting lactoferrin and lysozyme from pAF, we showed that there was a direct involvement of lysozyme and lactoferrin in the antibacterial activity of pAF. However, the depletion of these proteins without abolishing the antibacterial activity of pAF indicates that additional antibacterial factors are involved in the antibacterial activity of pAF.

\section{Conclusion}

The results of this study indicate that even after the removal of the insoluble components of human AF that the antibacterial activity of pAF is preserved and that a majority of the antibacterial activity of AF is contained within the soluble fraction. As we move towards controlled clinical studies to investigate the efficacy of using pAF in patients with burns and wounds, the antibacterial proteins identified under this study and their expression levels will be valuable in helping to assess their contributions in clinical outcome. In conclusion, pAF is rich in host defense proteins in which some are known for their 
antibacterial, antifungal, antiviral, anti-parasitic, antiinflammatory and immunomodulatory activities.

\section{Authors' contributions}

All authors were provided copies of the manuscript for their review. YM: study design, performed assays, analyzed and interpreted data, and helped to write the manuscript. JP: collection of birth tissue. AS-V: performed assays. MB: reviewed donor acceptance criteria. JK: study design. JAR: study design, analyzed and interpreted data and helped to write the manuscript. All authors read and approved the final manuscript.

\section{Author details}

${ }^{1}$ New Jersey Center for Biomaterials, Rutgers University, 145 Bevier Rd., Piscataway, NJ 08854, USA. ${ }^{2}$ Department Hematology \& Hematologic Malignancies, University of Utah, Salt Lake City, USA. ${ }^{3}$ Cell Therapy and Regenerative Medicine Facility, University of Utah, 676 Arapeen Drive, Suite 300, Salt Lake City, UT 84108, USA.

\section{Acknowledgements}

This study was supported by the Cell Therapy and Regenerative Medicine Facility at University of Utah and by the New Jersey Center for Biomaterials. The authors thank Eric Benedetti, Emily Peterson, Amber Preslar, Macon Latimer, Thalia Castro and Amit Singal for their technical supports. The authors would also like to thank Dr. John Phillips for his critical review of this manuscript.

\section{Competing interests}

The authors declare that they have no competing interests.

\section{Availability of data and materials}

The datasets during and/or analyzed during the current study available from the corresponding author on reasonable request.

\section{Consent for publication}

Not applicable.

\section{Ethics approval and consent to participate}

Donor consent, screening and infectious disease testing of human amniotic fluid (AF) were obtained under IRB\#36454 at the University of Utah.

\section{Funding}

This study was funded in part by the Cell Therapy and Regenerative Medicine Facility.

\section{Publisher's Note}

Springer Nature remains neutral with regard to jurisdictional claims in published maps and institutional affiliations.

Received: 19 December 2018 Accepted: 21 February 2019 Published online: 01 March 2019

\section{References}

1. Silini $A R$, et al. The long path of human placenta, and its derivatives, in regenerative medicine. Front Bioeng Biotechnol. 2015;3:162.

2. Tyszkiewicz JT, et al. Amnion allografts prepared in the central tissue bank in Warsaw. Ann Transplant. 1999;4(3-4):85-90.

3. Stock SJ, et al. Natural antimicrobial production by the amnion. Am J Obstet Gynecol. 2007;196(3):255.

4. Underwood MA, Gilbert WM, Sherman MP. Amniotic fluid: not just fetal urine anymore. J Perinatol. 2005;25(5):341-8.

5. Bergman N, Bercovici B, Sacks T. Antibacterial activity of human amniotic fluid. Am J Obstet Gynecol. 1972;114(4):520-3.

6. Cattaneo P. Potere Lisimico del liquido amniotico postere antilizimico del meconoio richerche superimentoli. Clin Obstet Gynaecol. 1949;51:60
7. Ojo VA, Okpere EE, Obaseiki-Ebor EE. Antimicrobial properties of amniotic fluid from some Nigerian women. Int J Gynaecol Obstet. 1986;24(2):97-101.

8. Akinbi HT, et al. Host defense proteins in vernix caseosa and amniotic fluid. Am J Obstet Gynecol. 2004;191(6):2090-6.

9. Mussap $M$, et al. Predictive value of amniotic fluid cystatin C levels for the early identification of fetuses with obstructive uropathies. BJOG. 2002;109(7):778-83.

10. Ammons MC, Copie V. Mini-review: Lactoferrin: a bioinspired, antibiofilm therapeutic. Biofouling. 2013;29(4):443-55.

11. Jasir A, et al. New antimicrobial cystatin C-based peptide active against Gram-positive bacterial pathogens, including methicillin-resistant Staphylococcus aureus and multiresistant coagulase-negative staphylococci. APMIS. 2003;111(11):1004-10.

12. Thadepalli H, Gangopadhyay PK, Maidman JE. Amniotic fluid analysis for antimicrobial factors. Int J Gynaecol Obstet. 1982;20(1):65-72.

13. Schlievert P, Johnson W, Galask RP. Bacterial growth inhibition by amniotic fluid. V. Phosphate-to-zinc ratio as a predictor of bacterial growth-inhibitory activity. Am J Obstet Gynecol. 1976;125(7):899-905.

14. Ismail MA, Salti Gl, Moawad AH. Effect of amniotic fluid on bacterial recovery and growth: clinical implications. Obstet Gynecol Surv. 1989;44(8):571-7.

15. Yung SC, Murphy PM. Antimicrobial chemokines. Front Immunol. 2012;3:276

16. Pierce J, et al. Collection and characterization of amniotic fluid from scheduled C-section deliveries. Cell Tissue Bank. 2016;17(3):413-25.

17. Leaper D, Assadian O, Edmiston CE. Approach to chronic wound infections. Br J Dermatol. 2015;173(2):351-8.

18. Costerton JW, Stewart PS, Greenberg EP. Bacterial biofilms: a common cause of persistent infections. Science. 1999;284(5418):1318-22.

19. Snyder RJ, et al. Wound biofilm: current perspectives and strategies on biofilm disruption and treatments. Wounds. 2017;29(6):S1-17.

20. Zhao R, et al. Inflammation in chronic wounds. Int J Mol Sci. 2016;17(12):2085.

21. Mao Y, Hoffman T, Johnson A, Duan-Arnold Y, Danikovitch A, Kohn J. Human cryopreserved viable amniotic membrane inhibits the growth of bacteria associated with chronic wounds. J Diab Foot Compl. 2016;8(2):23-30.

22. Mao Y, et al. Antimicrobial peptides secreted from human cryopreserved viable amniotic membrane contribute to its antibacterial activity. Sci Rep. 2017;7(1):13722.

23. Hicks RP, et al. Antimicrobial peptides containing unnatural amino acid exhibit potent bactericidal activity against ESKAPE pathogens. Bioorg Med Chem. 2013;21(1):205-14.

24. Warren GH, Gray J, Bartell P. The lysis of Pseudomonas aeruginosa by lysozyme. J Bacteriol. 1955;70(5):614-9.

25. Loukogeorgakis SP, De Coppi P. Concise review: amniotic fluid stem cells: the known, the unknown, and potential regenerative medicine applications. Stem Cells. 2017;35(7):1663-73.

26. Yoon BS, et al. Secretory profiles and wound healing effects of human amniotic fluid-derived mesenchymal stem cells. Stem Cells Dev. 2010;19(6):887-902.

27. Brzin J, et al. Human cystatin, a new protein inhibitor of cysteine proteinases. Biochem Biophys Res Commun. 1984;118(1):103-9.

28. Wittek N, Majewska E. Cystatin C-modulator of immune processes. Przegl Lek. 2010;67(7):484-7.

29. Leung-Tack J, et al. Neutrophil chemotactic activity is modulated by human cystatin C, an inhibitor of cysteine proteases. Inflammation. 1990;14(3):247-58.

30. Leung-Tack J, et al. Modulation of phagocytosis-associated respiratory burst by human cystatin C: role of the N-terminal tetrapeptide Lys-ProPro-Arg. Exp Cell Res. 1990;188(1):16-22.

31. Liao Y, et al. Proteomic characterization of human milk whey proteins during a twelve-month lactation period. J Proteome Res. 2011;10(4):1746-54.

32. Valenti P, Antonini G. Lactoferrin: an important host defence against microbial and viral attack. Cell Mol Life Sci. 2005;62(22):2576-87.

33. Puddu P, Valenti P, Gessani S. Immunomodulatory effects of lactoferrin on antigen presenting cells. Biochimie. 2009;91(1):11-8. 
34. Puddu P, et al. Bovine lactoferrin counteracts Toll-like receptor mediated activation signals in antigen presenting cells. PLOS ONE. 2011;6(7):e22504.

35. Schutyser E, Richmond A, Van Damme J. Involvement of CC chemokine ligand 18 (CCL18) in normal and pathological processes. J Leukoc Biol. 2005;78(1):14-26

36. Detmers PA, et al. Differential effects of neutrophil-activating peptide $1 /$ IL-8 and its homologues on leukocyte adhesion and phagocytosis. J Immunol. 1991:147(12):4211-7.
37. Aguila A, et al. Bacteriostatic activity of human lactoferrin against Staphylococcus aureus is a function of its iron-binding properties and is not influenced by antibiotic resistance. FEMS Immunol Med Microbiol. 2001;31(2):145-52.

38. Shimada T, et al. Staphylococcus aureus evades lysozyme-based peptidoglycan digestion that links phagocytosis, inflammasome activation, and IL-1beta secretion. Cell Host Microbe. 2010;7(1):38-49.
Ready to submit your research? Choose BMC and benefit from:

- fast, convenient online submission

- thorough peer review by experienced researchers in your field

- rapid publication on acceptance

- support for research data, including large and complex data types

- gold Open Access which fosters wider collaboration and increased citations

- maximum visibility for your research: over $100 \mathrm{M}$ website views per year

At BMC, research is always in progress.

Learn more biomedcentral.com/submissions 